\title{
HOT SURFACE IGNITION OF A COMPOSITE FUEL DROPLET
}

\author{
Dmitrii O. Glushkov ${ }^{\mathrm{a}}$, Pavel A. Strizhak, Ksenia Yu. Vershinina \\ National Research Tomsk Polytechnic University, 634050 Tomsk, Russia
}

\begin{abstract}
The present study examines the characteristics of conductive heating (up to ignition temperature) of a composite fuel droplet based on coal, liquid petroleum products, and water. In this paper, we have established the difference between heat transfer from a heat source to a fuel droplet in case of conductive (hot surface) and convective (hot gas) heat supply. The Leidenfrost effect influences on heat transfer characteristics significantly due to the gas gap between a composite fuel droplet and a hot surface.
\end{abstract}

\section{INTRODUCTION}

The use of composite fuels [1, 2], based on coal, liquid petroleum products, and water, is a promising way for rational use of fuel and energy sources. The main argument for the more widespread use of composite fuel in thermal power plants is the possibility to reduce the impact of emissions on the environment. Burning such fuel in thermal power plants causes less emission of ash, nitrogen, and sulfur oxide to the atmosphere, than burning coal or fuel oil. In addition, the operation of technological equipment may be improved due to the smaller amount of slag deposited on heating surfaces. Furthermore, composite fuels are fire and explosion safety, thus, their use may reduce the risk of accidents.

The use of composite fuels is one of the methods for solving the problems of rational resource management [3]. The operation of coal and petroleum enterprises is associated with the formation of a large number of low-grade wastes: oil sludge, the sifting of small coal fractions, coal slurry, and etc. As a result of technological processes, some waste is released into the environment, while some is stored and requires expensive disposal. However, oil and carbonaceous wastes from siftings and sludges, as well as low calorie coal may be used as a raw material for the preparation of composite fuels.

The aim of this work is to conduct evaluation experiments on the possibility to study the integrated characteristics of hot surface ignition of a composite fuel droplet.

\section{EXPERIMENTAL SETUP AND PROCEDURE}

Fig. 1 shows an experimental setup for the investigaitons of the regularities of physical and chemical processes at hot surface conductive heating of an organic water-carbon fuel droplet at a constant temperature. The base of a metal (steel) cylinder 10 (height $-70 \mathrm{~mm}$, diameter $-25 \mathrm{~mm}$ ) was a heating surface in our setup. An induction heater 1 (power consumption $15 \mathrm{kVA}$, operating frequency

\footnotetext{
${ }^{a}$ Corresponding author: dmitriyog@tpu.ru
} 
range $30-100 \mathrm{kHz}$, maximum current $22.5 \mathrm{~A}, 90 \%$ efficiency) heated the surface and maintained the desired temperature on the heating surface. One base of the cylinder 10 was set on the surface made from a heat resistant material. Another base was placed in the vicinity of a coil (an inductor) 9 made from a copper hollow tube. As a result of the alternating magnetic field, induced by the coil 9 , the material of the cylinder 10 was heated due to resistance and the eddy currents. The temperature of the heating surface was measured by thermocouple K 2 (the range of measured temperatures 273-1373 K, measurement error $\pm 3.3 \mathrm{~K}$ ) and an infrared pyrometer 3 (the range of measured temperatures 473$1773 \mathrm{~K}$, measurement error $1 \%$, resolution $1 \mathrm{~K}$, sighting index $1: 100$, emissivity setting range $0.01-$ 1.0, spectral range $8-14 \mu \mathrm{m}, \mathrm{RS}-232$ interface). The temperature was maintained at the desired level with the help of a controller 4 (the range of controlled temperatures $233-2473 \mathrm{~K}$, resolution $1 \mathrm{~K}$, RS232 interface), and the control algorithm of the induction heater 1. A chiller 5 (nominal cooling capacity $2.8 \mathrm{~kW}$, nominal air capacity $1000 \mathrm{~m}^{3} / \mathrm{h}$, the temperature in a water circuit 278-308 K) was used for water cooling of induction heater 1 power elements.

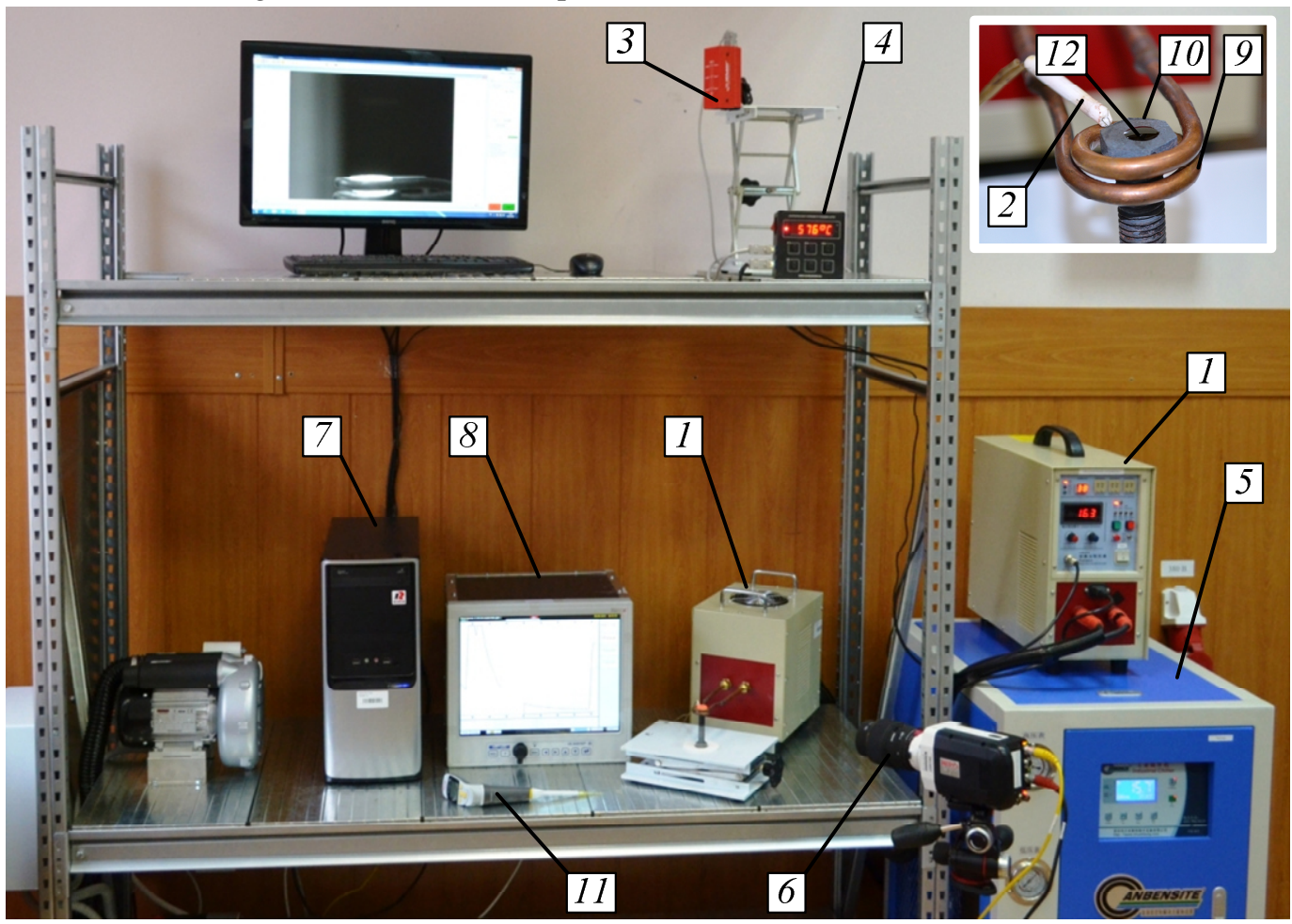

Figure 1. Experimental setup: 1 - induction heater; 2 - thermocouple; 3 - infrared pyrometer; 4 - temperature controller; 5 - chiller; 6 - high-speed video camera; 7 - personal computer; 8 - multi-channel technological register; 9 -coil; 10 - metal cylinder; 11 - pipette; 12 - composite fuel droplet.

We used a high-speed video camera "Phantom Miro M310" 6 (maximum speed $650000 \mathrm{fps,} \mathrm{full}$ resolution 1280x800, pixel size $20 \mu \mathrm{m}$, sensor size 25.6x16.0 mm, 12-bit depth, $1 \mu \mathrm{s}$ minimum exposure, 16 GB high-speed internal RAM) for video registration of investigated processes during the induction period. Video frames archives and temperature trends were recorded in the memory of a computer 7 (CPU frequency $3.4 \mathrm{GHz}$, Random Access Memory 8 GB, Read Only Memory 1 TB, LAN 10/100/1000 Mbit/s) and a multi-channel technological register RMT 59M 8 (12-channel analog input, Read Only Memory $2 \mathrm{~GB}$, the measurement range for thermocuples with a nominal static characteristic chromel-alumel 220-1570 K, measurement error $\pm 0.25 \%$, RS-232 interface), respectively. 
Organic water-carbon fuel droplets with a volume of $15 \mu$ were put on the heated surface by a dosing devise 11 (Finnpipette Novus: minimum dose volume $-5 \mu 1$, maximum dose volume $-50 \mu 1$, increment $-0.1 \mu \mathrm{l}$ ) with a special pipette tip. We put a droplet on the surface, when the temperature $T_{p}$ was stabilized at a given level (a deadband $\pm 5 \mathrm{~K}$ ). Analog processes of heat and mass transfer of liquid droplet during ignition at the heated substrate were inwestigated theoretically [4]. We calculated ignition delay times by analizing data from video recordings with a help of software "Tema Automotive". Experimental scattering data increase when the temperature of the heating surface decreaces. Therefore, the number of experiments at a fixed value $T_{p}$ was chosen taking into account this feature. The number of experiments ranged from 6 to 12 .

\section{Results and discussion}

At the initial stage, we performed the investigation of the ignition of one of the major fuel components - brown coal dust from Talovsky field with the size of particles $80 \mu \mathrm{m}$. When the surface temperature was more than $770 \mathrm{~K}$, the stable ignition of coal particles occurred (Fig. 2). Ignition delay time did not exceed $0.25 \mathrm{~s}$.
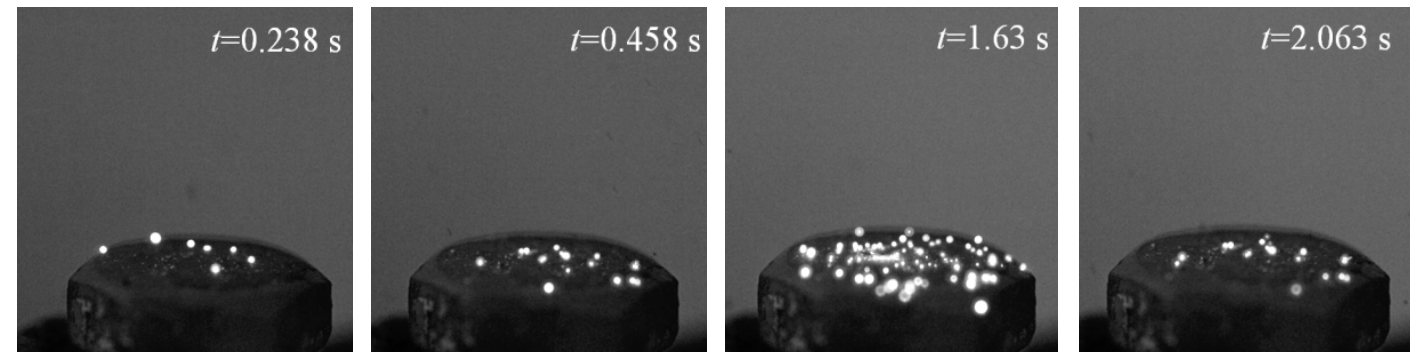

Figure 2. Hot surface ignition of brown coal dust at $T_{p}=770 \mathrm{~K}$.

In the next series of experiments, we chose hydrocarbon fuel as an investigated sample. We used water-carbon fuel, consisting from a mixture of water and fine brown coal. Fig. 3 shows the video recording frames of the interaction of a water-carbon fuel droplet with a surface, heated up to the temperature $770 \mathrm{~K}$. The results of the video analysis revealed the presence of the Leidenfrost effect, which manifests itself in the occurrence of steam layer between a liquid droplet and a heat source surface. When liquid evaporate intensively, the conditions of heat transfer with a hot surface deteriorate; drop is partially dispersed and slide from the surface. Hot surface ignition of coal particles occurs after at least $0.5 \mathrm{~s}$ from the time of the interaction of water-carbon fuel with an energy source.
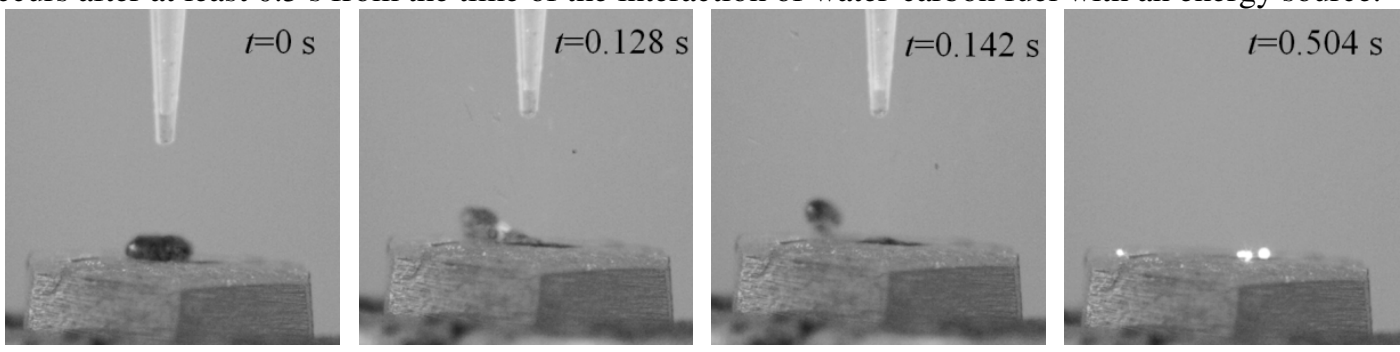

Figure 3. The interaction of a water-carbon fuel droplet with a heated surface at $T_{p}=770 \mathrm{~K}$.

When the temperature of the surface was equal to $380 \mathrm{~K}$, the impact of the Leidenfrost effect was minimal. It was observed (Fig. 4) a moderate evaporation of liquid. Coal dust particles remained on the heating surface after phase transition. Their ignition did not occur due to the low temperature of the surface. 

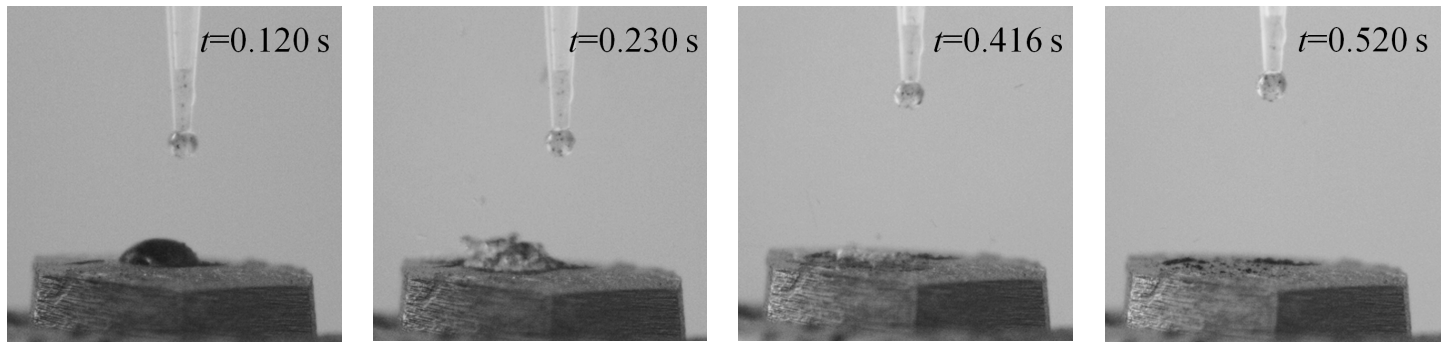

Figure 4. The interaction of a water-carbon fuel droplet with a heated surface at $T_{p}=380 \mathrm{~K}$.

In the last series of experiments, we have investigated the interaction of organic water-carbon fuel droplets, consisting of a mixture of water, brown coal particles with a size $80 \mu \mathrm{m}$ and a mineral oil, with a hot plate. The addition of the oil component to the fuel composition affects the characteristics of the interaction of droplets with the heat source compared to water-carbon composition.
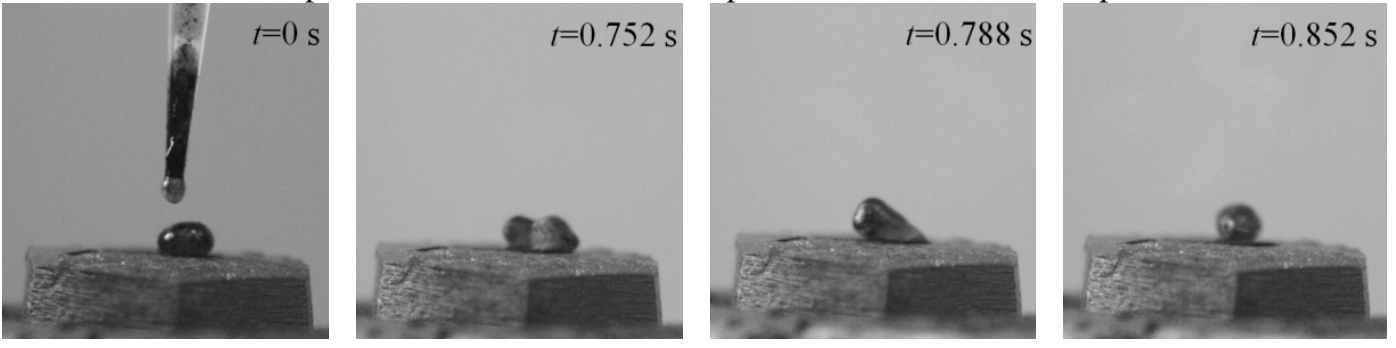

Figure 5. The interaction of an organic water-carbon fuel droplet with a heated surface at $T_{p}=520 \mathrm{~K}$.

When the temperature of the surface was equal to $520 \mathrm{~K}$, the significant deformation of the fuel droplet (Fig. 5) was observed without its sliding from the heating surface. It should be noted that with an increase in temperature of the heat source, similar process as in Fig. 3 took place.

\section{Conclusion}

Evaluation experiments showed a significant influence of Leidenfrost effect on the conditions of heat exchange at the hot surface conductive heating of composite fuel. In such conditions it was impossible to conduct experimental studies of physical and chemical characteristics that occur during ignition and combustion of fuel in boilers and furnaces.

The study was financially supported by the Russian Science Foundation (project No. 15-19-10003).

\section{References}

1. L.-J. Nie, Y. Liu, M. Huang, Adv. Mater. Res. 726, 1972 (2013).

2. X.-H. Fu, Z.-N. Wang, G. Novelli, B. de Benedetti, Journal of the China Coal Society 30, 493 (2005).

3. C.A. Kang, A.R. Brandt, L.J. Durlofsky, Energy 36, 6806 (2011).

4. D.O. Glushkov, P.A. Strizhak, Adv. Mech. Eng. 2014, 1 (2014). 